\title{
ARTICLE
}

\section{Soft Modelling of the Photolytic Degradation of Moxifloxacin Combining Surface Enhanced Raman Spectroscopy and Multivariate Curve Resolution}

\author{
Mónica Benicia Mamián-López ${ }^{1 *}$ \, Ronei J. Poppi² iD† \\ ${ }^{1}$ Universidade Federal do ABC, Av. dos Estados, 5001, Prédio B, Sala 1052. 09210-580, Santo André, SP, Brazil \\ ${ }^{2}$ Universidade Estadual de Campinas, Rua Josué de Castro, s/n, Cidade Universitária, 13083-970, Campinas, \\ SP, Brazil
}

† In memoriam (1961 - 2020)

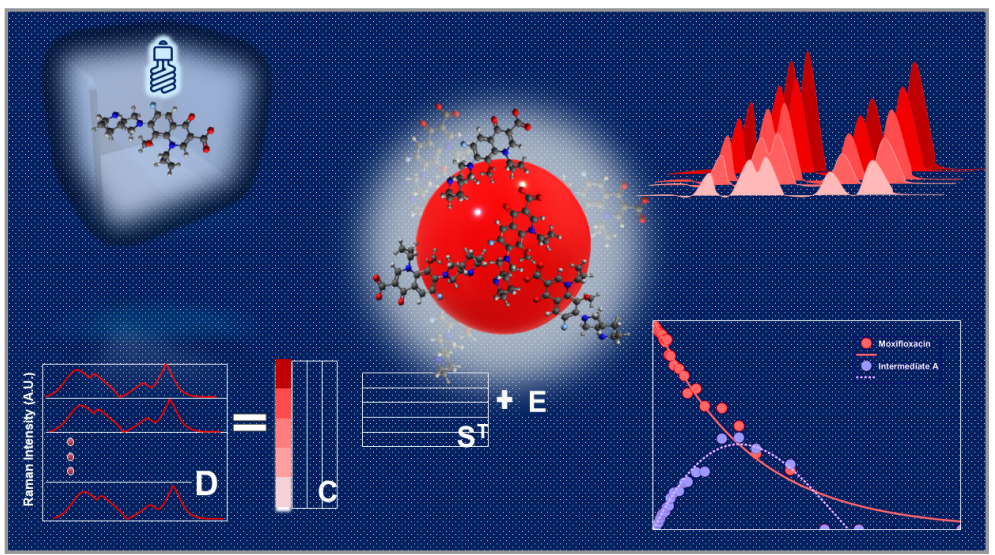

The antibiotic moxifloxacin had a recent surge in its use due to its broad spectrum of activity. However, due to the low metabolization inside the organism, it became an environmental concern. Here, the photolytic degradation of moxifloxacin antibiotic in alkaline medium was carried out and monitored through Surface enhanced Raman spectroscopy (SERS). Multivariate curve resolution method was applied to extract quantitative and kinetic information about the whole process, using correlation constraint to simultaneously quantify the variation of moxifloxacin concentration. The results showed that the photolysis follows an apparent first order kinetics with half-life of $47.5 \mathrm{~min}$. Also, SERS spectrum along with the calculated Raman spectra suggest that cleavage of the diazabicyclonyl substituent is the preferred photodegradation pathway, in agreement with previous reports.

Keywords: Chemometrics, Multivariate Curve Resolution, SERS spectroscopy, Gold Nanoparticles

\section{INTRODUCTION}

Moxifloxacin is a fourth-generation synthetic antibiotic belonging to the fluoroquinolone family. Its use has expanded recently due to its broad spectrum of activity, mainly towards gram-positive bacteria compared to other synthetic quinolones. It is used principally to treat pneumonia, bacterial sinusitis, and bronchitis. Furthermore, it can be administrated via intravenous, oral, and topical [1]. One of its characteristics is that it is not fully metabolized when used in medical treatments, being more of $50 \%$ excreted in its pharmaceutically active form. Due to its limited biodegradability and its increasing use, the research for efficient degradation 
methodologies of this kind of compounds has become a chief concern. Hence, studying its degradation pathways, products, and intermediate species is critical for searching for new methods.

Advanced oxidation processes (AOPs) include many techniques such as sonication, photocatalysis, ozonation, photolysis, among others, which are perhaps the most popular methods applied for environmental remediation [2-4]. Degradation of moxifloxacin has been studied [5] through photocatalytic and photolytic processes in aqueous medium [6] and in organic solvent [7] In both cases the degradation was monitored via chromatographic techniques and mass spectrometry.

The introduction of spectroscopic techniques to these studies provides a very complete structural landscape of the process, associated with high analytical sensitivity, and more straightforward experimental procedures, not requiring sample treatment and time-consuming stages before instrumental analysis. One of the more suitable techniques to reach these conditions is surface-enhanced Raman spectroscopy (SERS), which drastically intensifies the Raman scattering signal, providing vibrational fingerprint information. Its performance in aqueous (even biological) medium is optimal and requires, in most of cases, minimal experimental procedures before spectroscopic analysis. Although reproducibility has been a major issue in its application for quantitative purposes and researchers have been reluctant for several years about applying it in analytical studies, the scientific literature has already shown that quantifications are successful, and today, SERS spectroscopy is considered an analytical technique itself.

In a standard SERS analysis, the analyte of interest should be adsorbed onto a noble metal nanostructured surface, generally synthesized following a controlled reduction reaction of a salt of gold, silver, or copper. This way, a colloidal suspension containing nanoparticles with a given morphology is obtained. Then the next step includes the addition of an aliquot of the sample before to the spectral acquisition. Several variations of this procedure are widely available and allow analysis accordingly to the sample-surface system's chemical nature.

SERS spectra are typically information-rich eventually, even with overlapped signals. Since high volume of spectral data can be obtained quickly, computational methods for processing this data are mandatory. Still, nowadays, data processing and information extraction have an increasing role in research design and execution [8]. Usually, a first approach to the data sets is through exploratory methods such as Principal Component Analysis (PCA), aimed to reduce dimensionality of a data matrix by calculating new matrices of scores and loadings, projected on a new set of coordinates or principal components (PCs). Thus, trends or patterns can be easily visualized and then associated with their corresponding variables, responsible for a given behavior.

Decomposition methods are handy when the problem under study represents a mixture of components or changing processes [9]. The Multivariate Curve Resolution - Alternating Least Squares (MCR-ALS) method has become an exciting option when studying this kind of data, and the number of applications to truly diverse mixture problems has increased strikingly in recent years. Here, the original dataset, D, is decomposed into a bilinear equation containing the profiles of the "purest" components in a matrix $\mathbf{S}^{\top}$ and its respective relative concentrations in a matrix $\mathbf{C}$, following Equation 1. The $\mathbf{E}$ object is a residual matrix.

$$
D=C S^{T}+E
$$

In MCR-ALS, $\mathbf{S}^{\top}$ and $\mathbf{C}$ matrix are calculated iteratively using an initial estimation of pure signals (either calculated or acquired experimentally). Constraints related to the system being studied, such as nonnegativity of concentrations or signals, closure, or correlation constraints, can be applied, to provide chemical meaning to the calculated profiles and to minimize rotational ambiguity in the $\mathbf{C}$ and $\mathbf{S}^{\top}$ calculation. This chemometric tool has been successfully used for modelling kinetic processes [10,11], and calculating kinetic parameters. In this work, we have combined the high amount of structural information stored in SERS spectra and its ability to reach extremely low limit of detection values (values in the femtomolar regime has been reported [12]), with powerful chemometric tools to model and study the possible intermediates compounds in the photolytic degradation of moxifloxacin. In addition, the calculated Raman spectrum of 
moxifloxacin along with its observed SERS spectra allowed us to perform a band assignment which, to the authors knowledge, is being reported for the first time in this work.

\section{MATERIALS AND METHODS \\ Reagents and Solutions}

Chloroauric acid $\left(\mathrm{HAuCl}_{4}\right) 30 \%$ wt was purchased from Sigma-Aldrich, and Moxifloxacin hydrochloride was acquired from EMS-Brazil. Other reagents used, such as sodium citrate, sodium phosphate, sodium acetate, and sodium chloride were purchased from Synth. All solutions were prepared in Milli-Q water.

\section{Synthesis of the SERS substrate}

Colloidal Gold nanoparticles in suspension (AuNPs) was used as enhancing surface. The synthesis procedure was a modification of the Lee-Meisel method $[13,14]$, where a $\mathrm{HAuCl}_{4}$ solution was initially heated with constant stirring until boiling. Then, a preheated solution of sodium citrate was added to initiate the gold reduction to produce semi-spheric AuNPs $(\approx 60 \mathrm{~nm})$.

\section{Photolysis}

Stock solutions of moxifloxacin $\left(200.00 \mu \mathrm{g} \mathrm{mL}^{-1}\right)$ were prepared in $\mathrm{pH}$ values below, higher and between the two pKa values associated with its protonated and deprotonated forms ( $\mathrm{pKa}=5.7-9.2)$ to evaluate optimal condition favoring the degradation process. Thus, photolytic reactions were run at $\mathrm{pH}=4.5$ (acetate buffer), $\mathrm{pH}=6.5$ (not buffered) e $\mathrm{pH} 10.20$ (phosphate buffer). Four different initial concentrations were also tested $\left(20.00,15.00,10.00\right.$, and $\left.7.00 \mu \mathrm{g} \mathrm{mL}^{-1}\right)$.

The photolysis experiments were carried out in a closed chamber, temperature-controlled bath, as, where $200 \mathrm{~mL}$ of moxifloxacin solution (at the given $\mathrm{pH}$ ) was put in a Petri dish (of diameter equal to 12 $\mathrm{cm}$ ) during a total time of $2 \mathrm{~h}$, taking aliquots at reaction times of 0.60 and 120 minutes. A UV-C (16 W, $100-280 \mathrm{~nm}$ with main peak at $254 \mathrm{~nm}$ ) was used as a light source.

The optimal condition for the photolysis reaction ( $\mathrm{pH}=10.20$ and $10 \mu \mathrm{g} \mathrm{mL}^{-1}$ of initial concentration) was monitored for 8 hours, taking 26 aliquots of $400 \mu \mathrm{L}$ each, successively extracted according to Table I, and stored away from light. The process was run in triplicate and all samples were analyzed on the same day. Additionally, a control experiment was run simultaneously without exposition to UV-C light, and SERS spectra were registered before and at the end of the experiments. AuNPs were not part of the photolytic degradation, instead, they were used as enhancing surface for acquiring SERS spectra.

Table I. Aliquots acquisition time from photolytic process

\begin{tabular}{|l|l|l|l|l|l|l|l|l|l|l|l|l|l|l|}
\hline Aliquot (number) & 1 & 2 & 3 & 4 & 5 & 6 & 7 & 8 & 9 & 10 & 11 & 12 & 13 & $\ldots$ \\
\hline Time (min) & 0 & 1 & 2 & 3 & 4 & 5 & 6 & 7 & 8 & 9 & 10 & 12 & 15 & $\ldots$ \\
\hline
\end{tabular}

\begin{tabular}{|l|l|l|l|l|l|l|l|l|l|l|l|l|l|l|l|}
\hline Aliquot (number) & $\ldots$ & 14 & 15 & 16 & 17 & 18 & 19 & 20 & 21 & 22 & 23 & 24 & 25 & 26 & 27 \\
\hline Time (min) & $\ldots$ & 18 & 20 & 25 & 30 & 40 & 50 & 60 & 80 & 100 & 120 & 180 & 240 & 360 & 480 \\
\hline
\end{tabular}

\section{Standard solutions for the analytical curve}

Seven moxifloxacin standard solutions were prepared in triplicate between 0 - $12 \mu \mathrm{g} \mathrm{mL}^{-1}$ (in pH 10.20), and analytical curves were built from its SERS spectra. 


\section{Spectroscopic analysis}

Volumes of $200 \mu \mathrm{L}$ of each aliquot were added to $1.00 \mathrm{~mL}$ of AuNPs in a quartz cuvette and spectra were collected with six exposures of $8 \mathrm{~s}$ each and $250 \mathrm{~mW}$ of laser power (100\%). The spectral region used was between 200 and $2000 \mathrm{~cm}^{-1}$, with $4.0 \mathrm{~cm}^{-1}$ of spectral resolution.

The spectrometer was a Perkin Elmer Raman Station 400F, equipped with a $785 \mathrm{~nm}$ laser source, an Echelle grating, and a temperature-controlled $\left(-50^{\circ} \mathrm{C}\right)$ charged coupled device detector. The same conditions were used for all SERS spectra shown in this work.

\section{Band assignment and Raman spectrum calculations}

SERS bands attribution was based mainly on quantum chemistry calculations of the Raman spectrum. Furthermore, we compared our SERS spectra to those previously reported of similar fluoroquinolones (ofloxacin, enrofloxacin and ciprofloxacin) $[15,16]$ from the literature.

Quantum chemistry calculations were done using the Orca 4.2.1 package for a single moxifloxacin molecule using a conductor-like polarizable continuum model to mimic water solvation. The calculations were done using the M062X functional with the D3 Grimme dispersion correction and basis set def2TZVP(-f), following previous results from the literature for other fluoroquinolones [17-20].

\section{Data processing and multivariate analysis}

Data processing was performed in Matlab 7.8. Pre-processing was applied in PLS Toolbox 8.0 from Eigenvector. MCR-ALS analysis were run on MCR Toolbox [21] available at http://www.mcrals.info/.

Experimental data were arranged in matrix form with samples (SERS spectra from photolysis process) in the rows and variables (wavenumber, $\mathrm{cm}^{-1}$ ) in the columns. For the PCA analysis, spectra were normalized, the baseline was corrected with WLS (Automatic Weighted Least Squares) filter and mean centered. For MCR-ALS, the data was neither normalized nor mean centered. For quantification, the data was arranged as augmented matrix, correlation constraint was applied and a closure constraint for concentrations was also included (see Multivariate Curve Resolution: modelling and quantification section below).

\section{Quantification and assessment of Figures of Merit}

A pseudo-univariate model was built by plotting the real concentration values from each standard against relative concentrations recovered from MCR-ALS calculation (values in C matrix). Accordingly, figures of merit were estimated as proposed in literature [22,23]. The Limit of Detection (LOD) was calculated using the Equation 2:

$$
L O D=+t \frac{S_{R}}{b} \sqrt{\frac{1}{m}+\frac{1}{n}+\frac{(n-\bar{y})^{2}}{b^{2}\left(c_{i}-\bar{c}\right)^{2}}}
$$

where, $\mathrm{b}$ is the slope of the curve, $\mathrm{m}$ and $\mathrm{n}$ are the number of replicates and standards, respectively.

The concentration for the $i_{\text {th }}$ component and the mean concentration standard are $c_{i}$ and $\bar{c}$, respectively, and $\bar{y}$ is the mean predicted response (estimated by the model). The value $t$ corresponds to the Student value for $n-2$ degrees of freedom. The precision, $S_{R}$ was calculated through the Equation 3:

$$
S_{R}=\sqrt{\frac{\sum_{i=1}^{n}\left(\hat{\mathrm{y}}-y_{i}\right)^{2}}{n-1}}
$$

where $\hat{y}$ and $\mathrm{y}_{i}$, are the predicted and experimental response for the standard $i$, respectively. 
The Limit of Quantification (LOQ) value was calculated following the Equation 4:

$$
\mathrm{LOQ}=3.3 \mathrm{LOD}
$$

Finally, a test of significance for lack-of-fit $\left(\mathrm{F}_{L O F}\right)$ of the model was run using the unexplained variance and pure error. The $F_{L O F}$ calculated was compared to $F$ critical $\left(F_{c}\right)$ to estimate the lack of fit to the linear behavior.

\section{RESULTS AND DISCUSSION}

\section{Spectroscopic profile and band assignments}

Structurally, the moxifloxacin $\left(\mathrm{C}_{21} \mathrm{H}_{24} \mathrm{FN}_{3} \mathrm{O}_{4}\right)$ has a quinolone skeleton (drawn in Figure 1 with its atoms in blue), a fluorine atom at position 6, a methoxy group at position 8 and a diazabicyclonyl group at position 7, as shown in Figure 1 along with its SERS spectrum.

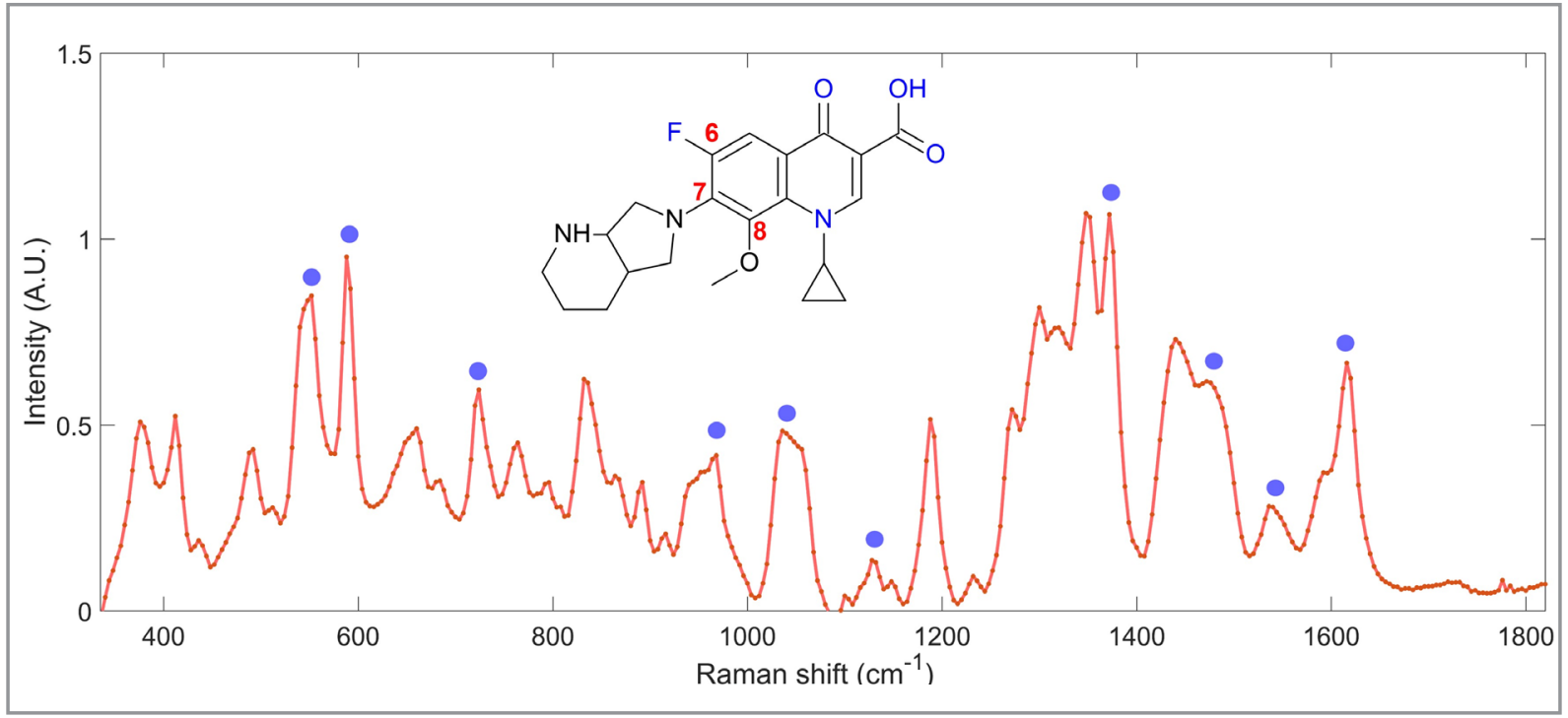

Figure 1. SERS spectrum of moxifloxacin $\left(10 \mu \mathrm{g} \mathrm{mL}^{-1}\right)$ in aqueous solution, on AuNPs and main band signals. Inset plot: molecular structure of moxifloxacin.

\section{Band assignments}

The main SERS features shows that diazabicyclonyl substituent has an important contribution to the whole spectra. Carbonyl and carboxyl modes are located at 724 and $1536 \mathrm{~cm}^{-1}$, the $-\mathrm{C}-\mathrm{O}$ bond of methoxy group contributes at $1100 \mathrm{~cm}^{-1}$ and the $-\mathrm{C}-\mathrm{F}$ bond has a small contribution at $1128 \mathrm{~cm}^{-1}$. A more complete description of other vibrational modes is presented in Table II.

Table II. SERS bands observed and calculated (Raman) for deprotonated moxifloxacin. Contributions of diazabicyclonyl group are marked with an asterisk $\left({ }^{*}\right)$.

\begin{tabular}{lll}
\hline SERS $\left(\mathbf{c m}^{-1}\right)$ & Calculated $\left(\mathbf{c m}^{-1}\right)$ & Vibrational modes description \\
\hline 552 & 551.84 & $\begin{array}{l}-\mathrm{CH}_{2} \text { twisting in diazabicyclonyl ring } \\
\text { Cyclopropyl ring deformation }\end{array}$ \\
588 & 596 & $\begin{array}{l}-\mathrm{CH}_{2} \text { rocking in diazabicyclonyl ring* } \\
\text { Quinolone ring deformation }\end{array}$ \\
\hline
\end{tabular}


Table II. SERS bands observed and calculated (Raman) for deprotonated moxifloxacin. Contributions of diazabicyclonyl group are marked with an asterisk $\left({ }^{*}\right)$. (Continuation)

\begin{tabular}{|c|c|c|}
\hline SERS $\left(\mathrm{cm}^{-1}\right)$ & Calculated $\left(\mathrm{cm}^{-1}\right)$ & Vibrational modes description \\
\hline 724 & 718.76 & $\begin{array}{l}-\mathrm{C}=\mathrm{O} \text { and }-\mathrm{O}-\mathrm{H} \text { bending } \\
-\mathrm{CH}_{2} \text { rocking of diazabicyclonyl ring and cyclopropyl } \\
\text { Quinolone ring deformation }\end{array}$ \\
\hline 944 & 945.96 & $-\mathrm{CH}_{2}$ stretch and twisting of diazabicyclonyl ring* \\
\hline 968 & 973.30 & $-\mathrm{CH}_{2}$ rocking of diazabicyclonyl ring* \\
\hline 1100 & 1100 & $\begin{array}{l}-\mathrm{CH}_{2} \text { wagging diazabicyclonyl ring and cyclopropyl } \\
\text { Stretch }-\mathrm{C}-\mathrm{O} \text { of methoxy group }\end{array}$ \\
\hline 1128 & 1125.43 & $\begin{array}{l}-\mathrm{CH}_{2} \text { rocking and wagging diazabicyclonyl ring* } \\
\text { Quinolone ring deformation } \\
-\mathrm{C}-\mathrm{F} \text { stretching }\end{array}$ \\
\hline 1232 & 1236.52 & $\begin{array}{l}-\mathrm{CH}_{2} \text { wagging of diazabicyclonyl ring * and cyclopropyl } \\
\text { Quinolone ring deformation }\end{array}$ \\
\hline 1372 & & $\begin{array}{l}-\mathrm{CH}_{2} \text { wagging of diazabicyclonyl ring * } \\
\text { Quinolone ring deformation }\end{array}$ \\
\hline 1436 & 1431 & $\begin{array}{l}-\mathrm{CH}_{2} \text { bending } \\
\text { Quinolone ring deformation } \\
-\mathrm{CH}_{2} \text { wagging of diazabicyclonyl ring* }\end{array}$ \\
\hline 1472 & 1471.77 & $-\mathrm{CH}_{2}$ bending of diazabicyclonyl ring* \\
\hline 1536 & 1529.4 & $\begin{array}{l}\text { Carbonyl and carboxyl stretch } \\
\text { In plane ring deformation }\end{array}$ \\
\hline
\end{tabular}

\section{Optimization of photolysis conditions}

Since $\mathrm{pH}$ plays an essential role in photolytic and photocatalytic reactions of fluoroquinolones [7] preliminary experiments at three different $\mathrm{pH}$ values were run (data not shown), to set the best condition to execute a rapid degradation, suitable for practical applications. Moxifloxacin showed to be highly stable at natural $\mathrm{pH}$ (less than $10 \%$ of the initial concentration was degraded in $2 \mathrm{~h}$ of process). About $25 \%$ of the initial moxifloxacin concentration reacted at $\mathrm{pH} 4.5$, with changes in the SERS spectrum obtained after $2 \mathrm{~h}$ of irradiation being evidenced by the decrease in correlation with the initial spectrum. Finally, alkaline condition $(\mathrm{pH}=10.20)$ showed to favor the degradation of $\approx 50 \%$ of the compound after 60 min of reaction, and this condition was chosen as optimal to study the kinetic process.

\section{Exploratory analysis}

SERS responses from the entire photolysis process were first explored using PCA analysis. The results allowed a more detailed visualization of how the compound(s) in the solution were changing with time as observed in Figure 2. Three main groups were observed along the reaction, with variables 756, 852, 828 and 1612 on PC1 dominating the first $80 \mathrm{~min}$. On PC2, the first $8 \mathrm{~min}$ are dominated by the variables at 904 and $1128 \mathrm{~cm}^{-1}$ (Loadings are included in Figure1SI). After $100 \mathrm{~min}$ of reaction, the spectra's intensity strongly decreases, only showing some SERS features of very low intensity (Figure 2SI).

The patterns observed in the scores plot show that SERS spectroscopy is a technique capable of observing and monitoring the entire chemical transformation (the photolysis degradation). It can give us insights into the initial reactant's structural changes, allowing us to infer possible intermediates. Furthermore, as we will argue ahead, it also allows assessing variations of their concentration. 


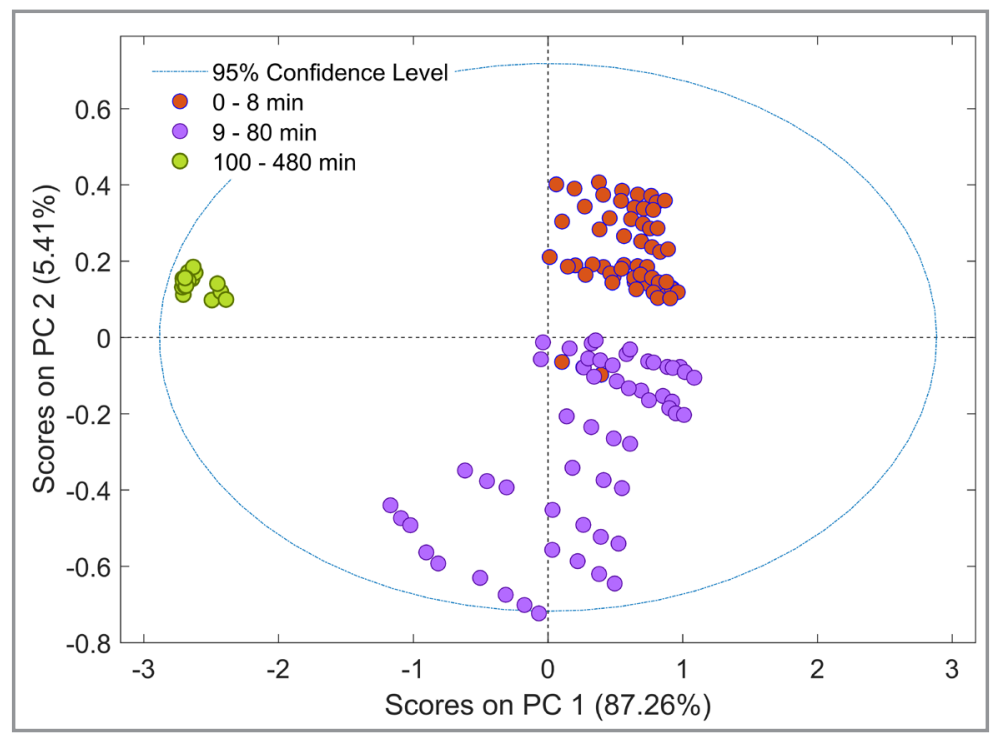

Figure 2. PCA scores plot for the SERS spectra of photolytic degradation of moxifloxacin in alkaline medium. Data were baseline-corrected and mean centered.

\section{Multivariate Curve Resolution: modelling and quantification}

As the objective of using MCR-ALS allied to SERS was both modelling the kinetic process and quantify the moxifloxacin degradation, the correlation constraint was chosen. This strategy has been successfully used [24] to address complex systems with overlapped signals and allows to reduce the range of feasible solutions.

To accomplish this, the SERS spectra acquired during the photolysis $\left(\mathbf{D}_{\mathbf{t}}\right)$ were arranged as a rowwise augmented matrix with the SERS spectra acquired for the analytical curve $\left(\mathbf{D}_{\mathrm{s}}\right)$. A constraint for concentrations was also introduced to run the MCR calculations, as depicted in Figure 3 . Briefly, a $\mathbf{C}_{\text {sel }}$ matrix containing the concentration values of the moxifloxacin standard solutions (first column of $\mathbf{C}_{\text {sel }}$ ) was included to force the final values in $\mathbf{C}$ (concentration profiles) matrix to be equal to the real values. The two remaining columns in $\mathbf{C}_{\text {sel }}$ are filled with zeros, since for their corresponding spectra (Ds), there is a unique contributor (moxifloxacin). The second calculation was performed by running the MCR-ALS method for the $D_{t}$ and $D_{s}$ matrix, separately. An analytical curve was tested but the results were not satisfactory. The arrangement for MCR-ALS calculation with constraint is shown in Figure 3.

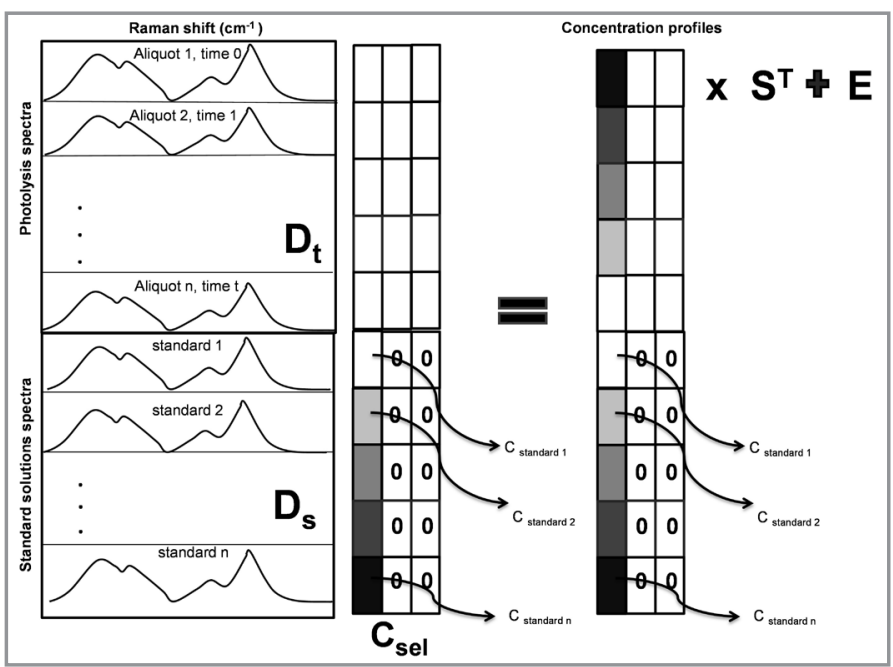

Figure 3. Data arrangement for MCR-ALS calculation with concentration constraint, including a $\mathbf{C}_{\text {sel }}$ matrix. 
The number of purest components (as calculated by the Singular Value Decomposition algorithm) was three, and, since where it is not possible to have the SERS responses of all species for the studied system an initial estimate was calculated by SIMPLISMA algorithm [25].

The relative concentrations in the $\mathbf{C}$ matrix, along with the concentrations from standard solutions, were used to build an analytical curve (Figure 3SI), for the quantitative assessment of changes in the moxifloxacin concentration. Plots for determining kinetic reaction order showed that the photolysis in alkaline medium follows an apparent first-order degradation model, as shown in Figure 4. Table II shows the reaction rate (for both approaches) estimated from the regression and the half-life calculation, following the Equation 5:

$$
t_{1 / 2}=\ln (2 / k)
$$

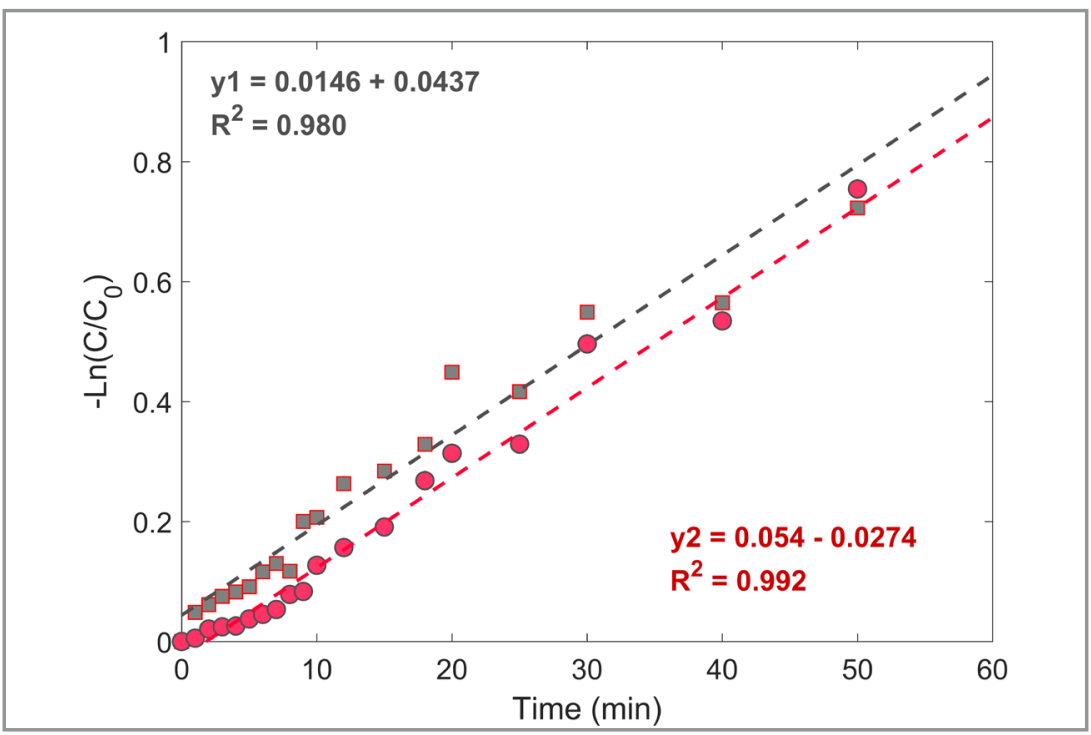

Figure 4. - $\mathrm{Ln}\left(\mathrm{C} / \mathrm{C}_{0}\right)$ vs. time $(\mathrm{min})$ for determining kinetic parameters, $k$ and $t_{1 / 2}$. Gray and red markers correspond to MCR-ALS without and with correlation constraint, respectively.

Table II. Reaction rate $(k)$ and half-life time $\left(t_{1 / 2}\right)$ for the studied compound with and without the augmented matrix correlation constraint

\begin{tabular}{lcc}
\hline MCR approach & $\boldsymbol{k}\left(\mathrm{min}^{-1}\right)$ & $\boldsymbol{t}_{1 / 2}(\mathrm{~min})$ \\
\hline $\begin{array}{l}\text { Augmented matrix and } \\
\text { correlation constraint }\end{array}$ & 0.0146 & 47.5 \\
$\begin{array}{l}\text { Without correlation } \\
\text { constraint }\end{array}$ & 0.0154 & 46.2 \\
\hline
\end{tabular}

These results follow the same trend as in previously reported studies for this reaction in similar conditions [6,7] where both strong pH dependence and apparent first-order kinetics were found. Relative concentrations in the $\mathbf{C}$ matrix also allowed to have an insight into the behavior of possible intermediates through the entire process. Plotting these values from MCR-ALS against time reaction provided a better landscape of the entire process, as shown in Figure 5. Here, the moxifloxacin loses about half its initial 
concentration at ca. $50 \mathrm{~min}$ while an intermediate $\mathrm{A}$ is being formed. A third possible compound, or intermediate B (showed in Figure 2SI) could be detected after 30 min of reaction, reaching a maximum value at around two hours, and becoming undetectable after this time of photolysis. After 100 min of reaction, SERS features were low and not quantifiable (Figure 3SI).

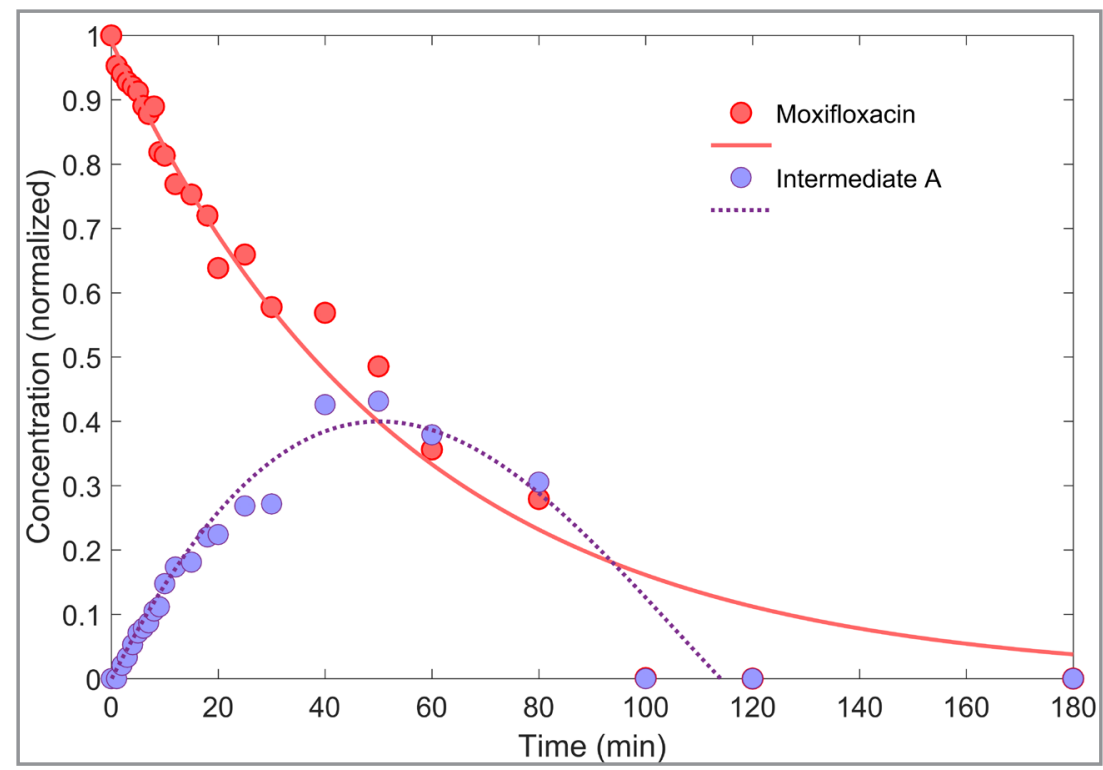

Figure 5. Photolytic degradation of moxifloxacin in $\mathrm{pH}=10.20$ and formation of a byproduct. Plot built with relative concentration values from $\mathbf{C}$ matrix, with augmented matrix and correlation constraint.

Although the spectral profiles recovered from the photolysis process have several common features, the behavior of the relative concentrations of moxifloxacin, intermediates $A$ and $B$ throughout the reaction (Figures 5 and 2SI) evolve very differently, thus suggesting that structural changes are occurring. Key regions, or bands, identified by comparing the different spectra (Figure 6), show an interesting correlation with the route proposed by Ahmad et al. [6] where three species could be identified following hydroxylation at the pyrrole ring, hydroxylation/oxidation of the side chain of piperidine ring, and finishing with a cleavage of the entire diazabicyclononane moiety. The vibrational modes changing through the photolysis in this work, i.e. 968, 1128, 1232, $1472 \mathrm{~cm}^{-1}$ are related to the diazabicyclonyl substituent, pointing to changes happening before cleavage. On the other hand, the band at $1536 \mathrm{~cm}^{-1}$, from carbonyl and carboxyl groups is almost disappears from moxifloxacin to intermediate $B$, which could be associated to a starting decarboxylation process. Also, subtle changes at $1128 \mathrm{~cm}^{-1}$, could be related to a possible cleavage of the -C-F bond (although this band also has contribution of the diazabicyclonyl group), but further experiments would be necessary to reach a conclusion.

It is interesting to notice that the bands related to the quinolone backbone remain strong through at least two hours, then there is a drastic lowering, becoming barely detectable through SERS. The absence of SERS features could indicate that, upon a possible cleavage of the substituent diazabicyclonyl, the remaining products lose their affinity towards AuNPs' surface. This implies, from the perspective of the interaction molecule-metallic surface, that the substituent moiety would be governing the whole molecule anchoring to the AuNPs, due to its more flexible nature, causing a favorable orientation to be observable in SERS. This dependence would also be associated to the marked separation of the groups observed in the scores plot discussed before (Figure 2). Evidence of further degradation was neither observed nor expected under our conditions. 


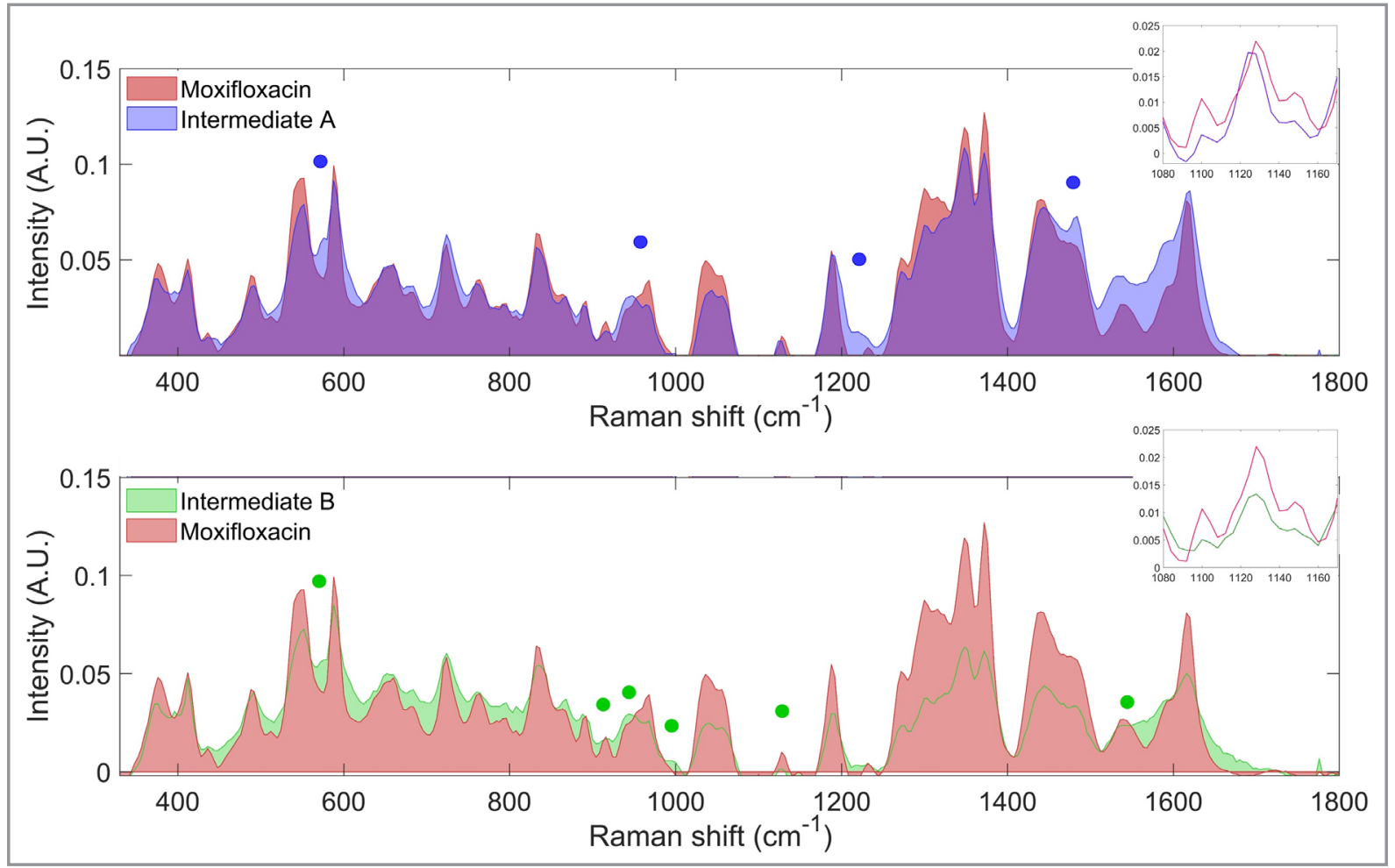

Figure 6. Spectral profiles calculated by MCR-ALS for the purest components of photolytic reaction of moxifloxacin and intermediates A and B). Inset plots: detail of the region between $1080-1170 \mathrm{~cm}^{-1}$.

\section{CONCLUSIONS}

In this work, a typical SERS experimental procedure was applied in the monitoring of a photocatalytic process, which offered information-rich spectroscopic information. Soft-modelling through MCR-ALS allowed to obtain quantitative information, and calculate kinetic parameters, despite that the system under study presents intermediates of the degradation process that are difficult to isolate, or even measure them using SERS, so that their profiles could be used as initial estimates for the MCR-ALS routine. The relative concentrations calculated by MCR-ALS method constrained with known information about the photolytic reaction allowed to simultaneously accompany the changes in the starting species and to model an intermediate product. Although there is few information available in the literature about the band assignments of moxifloxacin in SERS, the analysis of the calculated Raman spectrum allowed to suggest a degradation pathway that agreed with those previously reported, showing the photolytic cleavage of diazabicyclonyl group under alkaline conditions. Both the kinetic modelling and exploratory analysis suggested that the presence of the substituent played a role in the anchoring of moxifloxacin (or the intermediate products), towards the metallic surface, but additional experiments would be necessary to confirm this hypothesis.

\section{Acknowledgements}

This paper is dedicated to the memory of professor Ronei J. Poppi whom we lost in 2020. The authors acknowledge the "Instituto Nacional de Ciência e Tecnologia de Bioanalítica" (INCTBio) and the "Coordenação de Aperfeiçoamento de Pessoal de Nível Superior" (CAPES, Brasilia, Brazil) for fellowships and financial support. Also, the authors are indebted to Dr. Vitor Hugo Paschoal from the Institute of Chemistry at the University of São Paulo for the Raman spectrum calculations.

\section{Conflicts of interest}

The authors declare that there is no conflict of interest. 


\section{REFERENCES}

1. Scholar, E. "Moxifloxacin". In: xPharm: The Comprehensive Pharmacology Reference, Elsevier, 2007, pp 1-6.

2. Kanakaraju, D.; Glass, B. D.; Oelgemöller, M. J. Environ. Manage., 2018, 219, pp 189-207 (http:// dx.doi.org/10.1016/j.jenvman.2018.04.103).

3. Anjali, R.; Shanthakumar, S. J. Environ. Manage., 2019, 246, pp 51-62 (http://dx.doi.org/10.1016/j. jenvman.2019.05.090).

4. Khare, P.; Patel, R. K.; Sharan, S.; Shankar, R. Recent trends in advanced oxidation process for treatment of recalcitrant industrial effluents. In: Shah, M. (Ed.). Advanced Oxidation Processes for Effluent Treatment Plants, Elsevier, 2020, pp 137-160.

5. Yahya, M. S.; Beqqal, N.; Guessous, A.; Arhoutane, M. R.; El Kacemi, K. Cogent. Chem., 2017, 3 (1), 1290021 (http://dx.doi.org/10.1080/23312009.2017.1290021).

6. Ahmad, I.; Bano, R.; Musharraf, S. G.; Ahmed, S.; Sheraz, M. A.; ul Arfeen, Q.; Bhatti, M. S.; Shad, Z. AAPS PharmSciTech, 2014, 15 (6), pp 1588-1597 (http://dx.doi.org/10.1208/s12249-014-0184-x).

7. Van Doorslaer, X.; Demeestere, K.; Heynderickx, P. M.; Van Langenhove, H.; Dewulf, J. Appl. Catal., B., 2011, 101 (3-4), pp 540-547 (http://dx.doi.org/10.1016/j.apcatb.2010.10.027).

8. Langer, J.; de Aberasturi, D. J.; Aizpurua, J.; Alvarez-Puebla, R. A.; Auguié, B.; Baumberg, J. J.; Bazan, G. C.; Bell, S. E. J.; Boisen, A.; Brolo, A. G.; et al. ACS Nano, 2020, 14 (1), pp 28-117 (http://dx.doi. org/10.1021/acsnano.9b04224).

9. Bedia, C.; Sierra, À.; Tauler, R. Anal. Bioanal. Chem., 2020, 412 (21), pp 5179-5190 (http://dx.doi. org/10.1007/s00216-020-02595-8).

10. Gómez-Canela, C.; Bolivar-Subirats, G.; Tauler, R.; Lacorte, S. J. Pharm. Biomed. Anal., 2017, 137, pp 33-41 (http://dx.doi.org/10.1016/j.jpba.2017.01.019).

11. Kaur, P.; Imteaz, M. A.; Sillanpää, M.; Sangal, V. K.; Kushwaha, J. P. Chemom. Intell. Lab. Syst., 2020, 203, 104027 (http://dx.doi.org/10.1016/j.chemolab.2020.104027).

12. Indrasekara, A. S. D. S.; Meyers, S.; Shubeita, S.; Feldman, L. C.; Gustafsson, T.; Fabris, L. Nanoscale, 2014, 6 (15), pp 8891-8899 (http://dx.doi.org/10.1039/C4NR02513J).

13. Lee, P. C.; Meisel, D. J. Phys. Chem., 1982, 86 (17), pp 3391-3395 (http://dx.doi.org/10.1021/ j100214a025).

14. Mamián-López, M.; Temperini, M. Quim. Nova, 2019 (http://dx.doi.org/10.21577/0100-4042.20170424).

15. Hong, K. Y.; de Albuquerque, C. D. L.; Poppi, R. J.; Brolo, A. G. Anal. Chim. Acta, 2017, 982, pp 148155 (http://dx.doi.org/10.1016/j.aca.2017.05.025).

16. El-Zahry, M. R.; Lendl, B. Spectrochim. Acta Part A Mol. Biomol. Spectrosc., 2018, 193, pp 63-70 (http://dx.doi.org/10.1016/j.saa.2017.12.007).

17. Neese, F.; Wennmohs, F.; Becker, U.; Riplinger, C. J. Chem. Phys., 2020, 152 (22), 224108 (http:// dx.doi.org/10.1063/5.0004608).

18. Weigend, F. Phys. Chem. Chem. Phys., 2006, 8(9), pp 1057-1065 (http://dx.doi.org/10.1039/b515623h).

19. Weigend, F. J. Comput. Chem., 2008, 29 (2), pp 167-175 (http://dx.doi.org/10.1002/jcc.20702).

20. Weigend, F.; Ahlrichs, R. Phys. Chem. Chem. Phys., 2005, 7 (18), pp 3297-3305 (http://dx.doi. org/10.1039/b508541a).

21. Jaumot, J.; de Juan, A.; Tauler, R. Chemom. Intell. Lab. Syst., 2015, 140, pp 1-12 (http://dx.doi. org/10.1016/j.chemolab.2014.10.003).

22. Saurina, J.; Leal, C.; Compañó, R.; Granados, M.; Prat, M. D.; Tauler, R. Anal. Chim. Acta, 2001, 432 (2), pp 241-251 (http://dx.doi.org/10.1016/S0003-2670(00)01378-7).

23. Olivieri, A. C. Chem. Rev., 2014, 114 (10), pp 5358-5378 (http://dx.doi.org/10.1021/cr400455s).

24. Bayat, M.; Marín-García, M.; Ghasemi, J. B.; Tauler, R. Anal. Chim. Acta, 2020, 1113, pp 52-65 (http:// dx.doi.org/10.1016/j.aca.2020.03.057).

25. Windig, W.; Guilment, J. Anal. Chem., 1991, 63 (14), pp 1425-1432 (http://dx.doi.org/10.1021/ ac00014a016). 


\section{SUPPORTING INFORMATION}

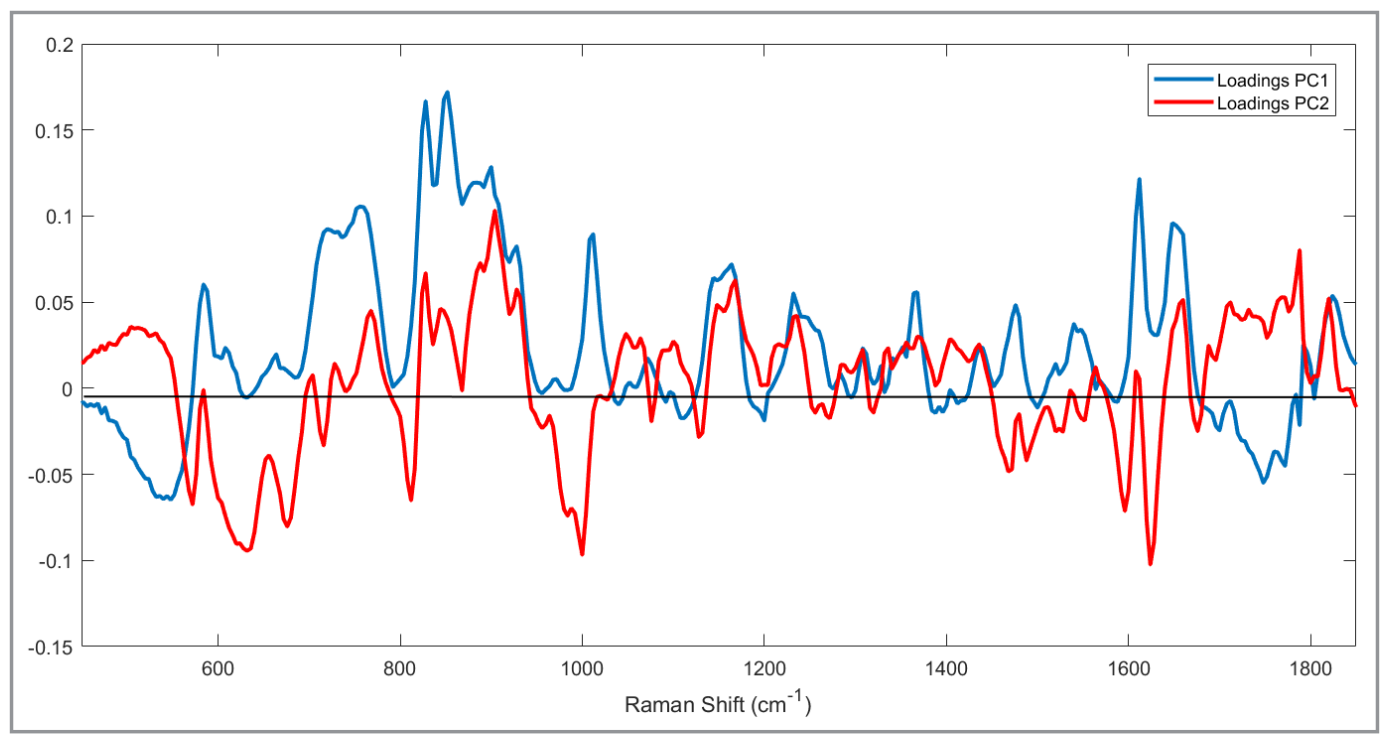

Figure 1SI. Loadings plot for PCA analysis of Figure 2 in main text.

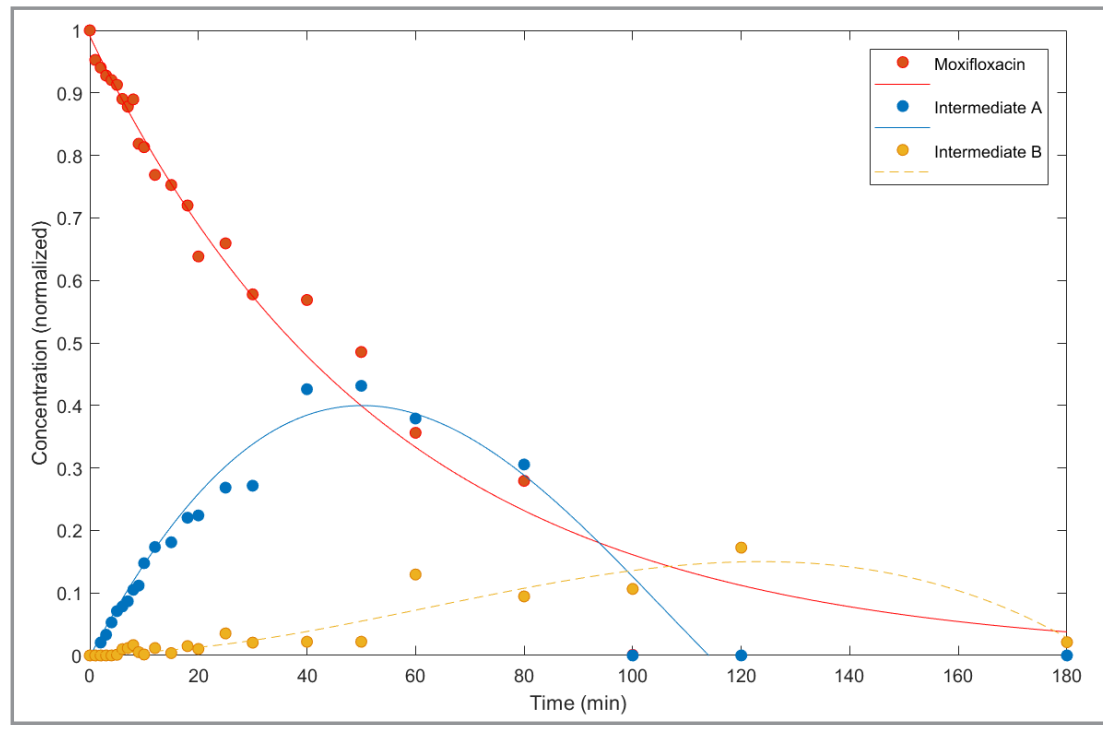

Figure 2SI. Photolytic degradation of moxifloxacin in $\mathrm{pH}=10.20$ and formation of a byproduct. Plot built with relative concentration values from $\mathbf{C}$ matrix, with augmented matrix and correlation constraint. 


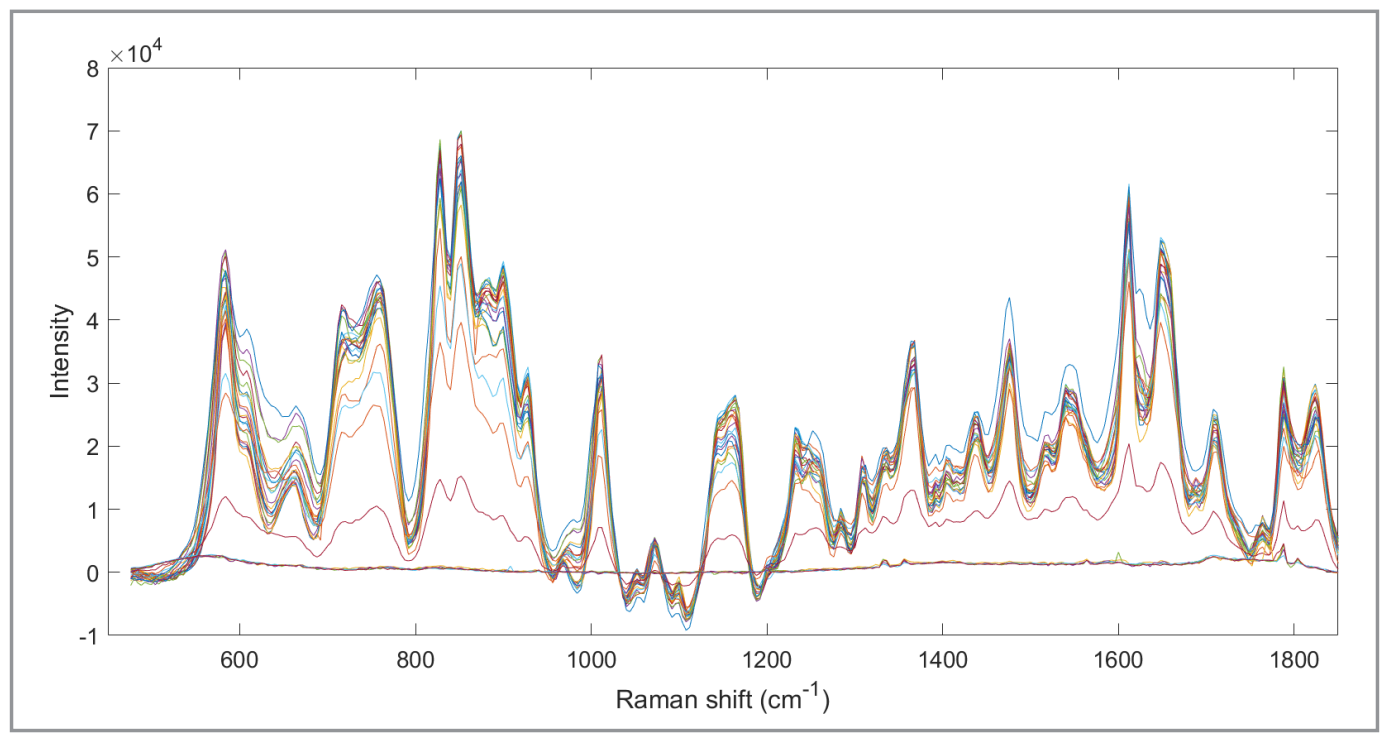

Figure 3SI. SERS Spectra through an entire photolytic process of moxifloxacin.

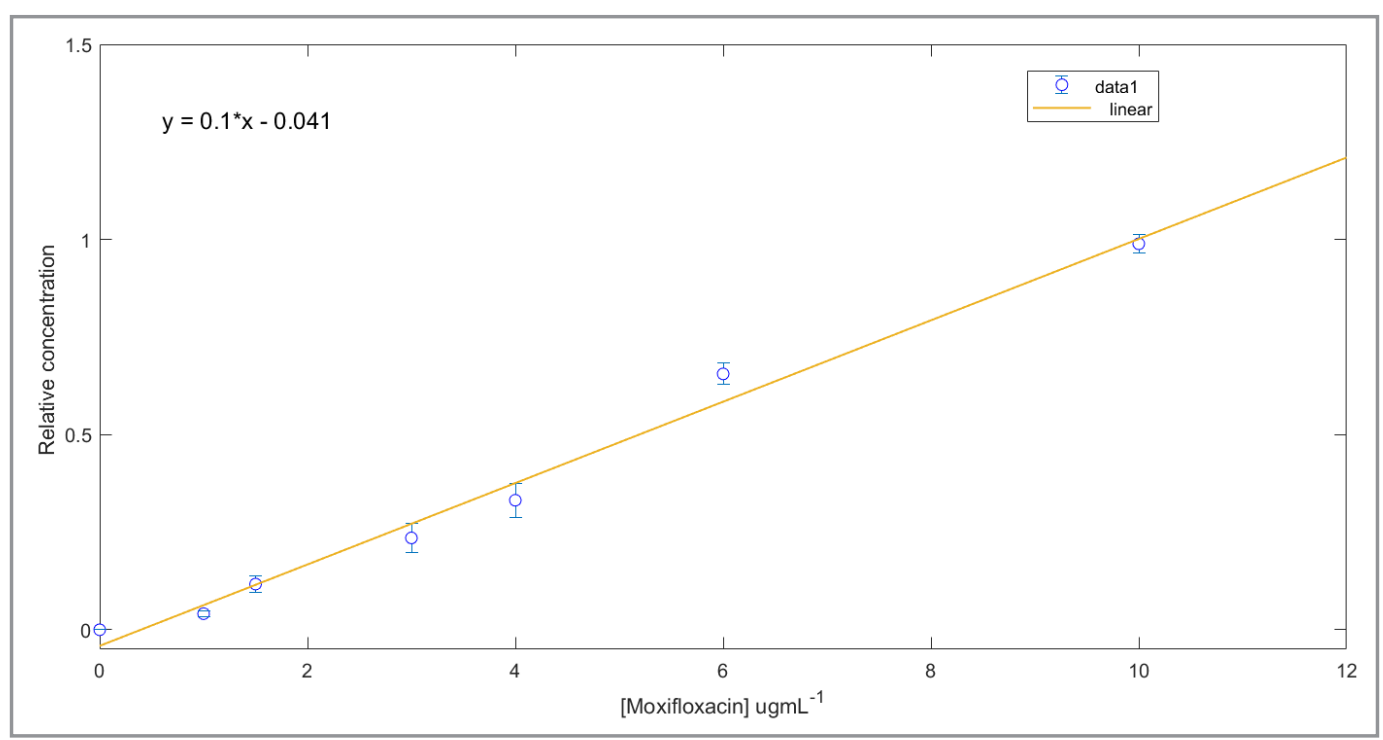

Figure 4SI. Analytical curve for the moxifloxacin quantification. Real concentration values of moxifloxacin standards vs. relative concentration values from MCR-ALS calculations.

Figures of Merit for Moxifloxacin quantification (Analytical Curve of Figure 3SI)

\begin{tabular}{cccc}
\hline $\mathbf{R}^{2}$ & $\operatorname{LOD}\left(\mu \mathrm{g} \mathrm{mL}^{-1}\right)$ & $\mathrm{LOQ}\left(\boldsymbol{\mu g ~ m L} \mathbf{~}^{-1}\right)$ & $\mathbf{F}_{\text {LOF }} / \mathrm{F}_{\mathrm{c}}$ \\
\hline 0.989 & 0.12 & 0.36 & $2.06 / 3.03$ \\
\hline
\end{tabular}

